\title{
ANAPHYLAXIS FOLLOWING UNNECESSARY MENINGOCOCCAL CHEMOPROPHYLAXIS OF A HEALTHCARE WORKER
}

\author{
F Giovanetti (fgiovanetti@aslcn2.it) ${ }^{1}$ \\ 1. Azienda Sanitaria Locale CN2 Alba Bra, Dipartimento di Prevenzione (Local Health Unit CN2 - Alba Bra, Department of \\ Prevention), Alba, Piedmont Region, Italy
}

We report a case of anaphylaxis following meningococcal chemoprophylaxis of a healthcare worker, despite no history of direct contact with a patient who had meningococcal disease. The public health implications of this case are discussed.

\section{Introduction}

Chemoprophylaxis of meningococcal disease is usually recommended for close contacts, such as household members and persons directly exposed to a patient's respiratory secretions, as these have been shown to have a higher risk of invasive meningococcal disease [1]. However, chemoprophylaxis is not recommended for low risk or indirect contacts, partly because of possible adverse outcomes. These include adverse events to prophylactic antibiotics, development of antibiotic resistance and eradication of non-pathogenic Neisseria species which may confer protection against Neisseria meningitidis [2,3]. In particular, administration of prophylactic antibiotics for healthcare workers is recommended only after an unprotected exposure to respiratory secretions of an index case. We describe a case of anaphylaxis following chemoprophylaxis of a healthcare worker with no history of direct contact with a patient who had sepsis caused by $N$. meningitidis. The public health implications of this case are discussed.

\section{Case description}

In March 2009, a woman in her 40s was admitted to a hospital in the Piedmont Region, Italy, with a one-day history of fever and myalgia. On admission, the patient was unconscious, with hypotension, tachycardia, acidosis and a truncal petechial rash. Clinical and laboratory features suggested a septic shock with disseminated intravascular coagulation. Her condition rapidly worsened and death occurred two hours after admission, despite resuscitation.

As the presumptive diagnosis was meningococcal disease, pathologists collected samples of blood, cerebrospinal fluid and petechial smears during the post mortem examination. PCR was subsequently performed at the Istituto Superiore di Sanità Infectious Diseases Laboratory in Rome, and N. meningitidis serogroup B was detected in all samples.

In accordance with the local public health unit, the hospital management recommended treatment with a single $500 \mathrm{mg}$ dose of ciprofloxacin for two doctors who had had unprotected exposure to the respiratory secretions of the patient. Chemoprophylaxis was also administered to three healthcare workers of the hospital staff. Outside the hospital setting, the contact tracing identified two household members as well as eight contacts who had been presumably exposed to the respiratory secretions of the patient and all took chemoprophylaxis. No secondary cases occurred in the following 30 days.

The following day, a healthcare worker in the same unit as one of the exposed doctors decided to take a single $500 \mathrm{mg}$ dose of ciprofloxacin for fear of contracting meningococcal disease, although she had no history of direct contact with the index case. On this basis, she had not been classified by the hospital management as a close contact and thus had not been offered chemoprophylaxis. Approximately 20 minutes after taking ciprofloxacin at the workplace, she was admitted to the emergency room with pharyngeal oedema, tongue swelling and generalised skin rash. The patient recovered gradually after administration of adrenalin, antihistamines and corticosteroids. She had used ciprofloxacin in the past without any adverse reaction.

\section{Discussion}

Invasive meningococcal disease is uncommon in Italy. Approximately 180 cases ( 0.3 per 100,000 population) are notified annually to the infectious diseases surveillance system [4]. The highest incidence is seen among children under five years old. In the Piedmont Region, an area in north-west Italy with 4.3 million inhabitants and active laboratory-based surveillance, the incidence appears to be higher: $0.4-0.7$ per 100,000 population, with a constant peak during the first year of life, ranging from five to six cases per year per 100,000 population [5].

Chemoprophylaxis is recommended in Italy only for persons with close contact to the index case up to one week before the onset of the patient's symptoms. Close contacts include: household members, contacts in child-care centres, and persons directly exposed to the patient's oral secretions [6]. Giving chemoprophylaxis to people who have not been in close contact with an index case has not proved to be effective in preventing secondary cases and is usually not recommended $[3,7]$.

In Italy, national guidelines on meningococcal chemoprophylaxis for healthcare workers are not available. Nevertheless, the regional health authorities as well as hospitals have developed standard operating procedures, usually based on international authoritative sources, such as the Centers for Disease Control and Prevention (CDC) in Atlanta, United States (US). In accordance with CDC guidance, the operating procedures of the local health unit involved in this case recommend chemoprophylaxis for healthcare workers 
after an unprotected airway exposure to infectious respiratory droplets within a distance of $1 \mathrm{~m}$ from a probable or confirmed case of meningococcal disease; this may happen typically during mouth-to-mouth resuscitation or management of an endotracheal tube $[6,8]$.

A study in the United Kingdom found an attack rate of 0.8 per 100,000 healthcare workers in close contact with cases of meningococcal disease, i.e. 25 times higher than in the general population [9]. The study identified three cases of meningococcal disease in healthcare workers during a period of 15 years: all had spent at least 30 minutes in contact with the index case immediately before or after hospital admission, all had been exposed to the patients' respiratory droplets, and none had used face shields and surgical masks or taken prophylactic antibiotics.

The fluoroquinolone ciprofloxacin is often used for meningococcal chemoprophylaxis in adults because it can be given as a single oral dose, is effective in eradicating meningococcal carriage and does not interact with oral contraceptives. For the same reasons, however, unnecessary chemoprophylaxis is more likely to occur with ciprofloxacin than with other prophylactic antibiotics. Rifampicin requires a total of four doses in the course of two days and can interfere with oral contraceptives; ceftriaxone is administered as a single dose, but is not popular because it can only be administered parenterally.

Anaphylaxis following ciprofloxacin administration has been described before. In particular, three cases of anaphylactoid reactions were reported after oral administration of $500 \mathrm{mg}$ ciprofloxacin to 3,200 students after two cases of meningococcal disease in the same university [10]. Limited data on the magnitude of allergic reactions following administration of drugs are available, mainly because clinical manifestations are heterogeneous (from mild to severe and potentially life-threatening) and furthermore some reactions suggesting an immunologic pathogenesis might be linked to a non-allergic mechanism. Likewise, the incidence of allergic reactions induced by oral antibiotics such as ciprofloxacin is difficult to estimate. However, according to a case/non-case study conducted on data from a passive adverse events surveillance programme, fluoroquinolones were associated with a significant increase in the reporting odds ratio of allergic reactions (2.09, 95\% confidence interval (Cl): 1.85-2.36) [11]. Moreover, an incidence of 5.4 (95\% Cl: 4.4-6.5) allergic reactions per 10,000 first administrations of ciprofloxacin has been derived from the database of a large health insurance company [12].

Another reason for concern is the potential development of antibiotic resistance. Three cases of meningococcal disease caused by a $N$. meningitidis serogroup $B$ strain resistant to ciprofloxacin were recently reported in the United States [13]. The widespread use of fluoroquinolones, which are commonly prescribed in the United States [14], and the consequent emergence of resistant strains may explain these findings.

Finally, prophylactic antibiotics can eliminate carriage of $N$. lactamica, which leads to cross-protective immunity against $N$. meningitidis and therefore may confer protection against meningococcal disease $[15,16]$. This concerns young children in particular, since carriage of $N$. lactamica is inversely related to age [16].

Quantitative data about the overuse of chemoprophylaxis are scarce. A study in the United Kingdom evaluated prescribing of chemoprophylaxis for contacts of meningococcal disease by general practitioners and hospital staff [17]. Prescribing by hospital doctors was consistent with official recommendations, whereas general practitioners prescribed $118 \%$ more chemoprophylaxis than recommended. Furthermore, the highest level of unrecommeded prescriptions was observed in regions where there were both high incidence rates and high levels of publicity surrounding the cases. Most likely, this inappropriate prescribing is client-driven because meningococcal disease raises anxiety among the involved population.

\section{Conclusions}

Use of prophylactic antibiotics against meningococcal disease can lead to potentially severe adverse events, development of antibiotic resistance and eradication of non-pathogenic Neisseria species that may elicit cross-protective immunity. Therefore, information should be provided to the public and to healthcare workers about the potential risks from indiscriminate use of prophylactic antibiotics. Available data should be used to compare the risks related to different patterns of exposure to a case of meningococcal disease versus the possible adverse outcomes of chemoprophylaxis. To this end, thorough counselling and communication of the population-based and individual-based risk represent a key factor in the public health management of meningococcal disease cases.

\section{References}

1. Purcell B, Samuelsson S, Hahne SJ, Ehrhard I, Heuberger S, Camaroni I, et al. Effectiveness of antibiotics in preventing meningococcal disease after a case: systematic review. BMJ. 2004;328(7452):1339.

2. Kristiansen $B E$, Knapsog $A B$. Secondary prevention of meningococcal disease. BMJ. 1996;312(7031):591-2.

3. Health Protection Agency Meningococcus Forum. Guidance for public health management of meningococcal disease in the UK. August 2006. Available from: http://www.hpa.org.uk/web/HPAwebFile/HPAweb_C/1194947389261

4. Sistema Informatizzato Malattie Infettive (SIMI) [Information system for infectious diseases]. Istituto Superiore di Sanità. Dati di sorveglianza sulle meningiti [Surveillance data on meningitis]. SIMI [Accessed 25 April 2009 ]. Available from: http://www.simi.iss.it/dati.htm

5. Barale A, Criveller T, Lombardi D. Sorveglianza di laboratorio delle infezioni batteriche invasive in Regione Piemonte. Risultati attività anno 2006. [Laboratory surveillance of invasive bacterial infections in Regione Piemonte. Activity results for the year 2006]. Available from: http://epidem.asl20. piemonte. $i t /$ ?sezione $=323$

6. Bilukha 00, Rosenstein N, National Center for Infectious Diseases, Centers for Disease Control and Prevention (CDC). Prevention and control of meningococcal disease: recommendations of the Advisory Committee on Immunization Practices (ACIP). MMWR Recomm Rep. 2005;54(RR-7):1-21.

7. American Academy of Pediatrics. Red Book 2006. Report of the Committee on Infectious Diseases. 27th ed. Elk Grove Village, IL: American Academy of Pediatrics 2006.

8. Bolyard EA, Tablan OC, Williams WW, Pearson ML, Shapiro CN, Deitchmann SD. Guideline for infection control in healthcare personnel, 1998. Hospital Infection Control Practices Advisory Committee. Infect Control Hosp Epidemiol. 1998;19(6):407-63.

9. Gilmore A, Stuart J, Andrews N. Risk of secondary meningococcal disease in health-care workers. Lancet. 2000;356(9242):1654-5.

10. Burke P, Burne SR. Allergy associated with ciprofloxacin. BMJ. 2000;320(7236):679.

11. Salvo F, Polimeni G, Cutroneo PM, Leone R, Confortic A, Moretti U, et al. Allergic reactions to oral drugs: A case/non-case study from an Italian spontaneous reporting database (GIF). Pharmacol Res. 2008;58(3-4):202-7.

12. Johannes CB, Ziyadeh N, Seeger JD, Tucker E, Reiter C, Faich G. Incidence of allergic reactions associated with antibacterial use in a large, managed care organisation. Drug Saf. 2007;30(8):705-13.

13. Wu HM, Harcourt BH, Hatcher CP, Wei SC, Novak RT, Wang X, et al. Emergence of ciprofloxacin-resistant Neisseria meningitidis in North America. N Engl J Med. 2009;360(9):886-92. 
14. Steinman MA, Gonzales R, Linder JA, Landefeld CS. Changing Use of Antibiotics in Community-Based Outpatient Practice, 1991-1999. Ann Intern Med. 2003;138(7):525-33.

15. Yazdankhah SP, Caugant DA. Neisseria meningitidis: an overview of the carriage state. J Med Microbiol 2004;53(Pt 9):821-32.

16. Bennet JS, Griffiths DT, Mc Carthy ND, Sleeman KL, Jolley KA, Crook DW, et al. Genetic diversity and carriage dynamics of Neisseria lactamica in infants. Infect Immun. 2005;73(4):2424-32.

17. Marks PJ, Neal KR. Variations in chemoprophylaxis for meningococcal disease: a retrospective case note review, analysis of routine prescribing data and questionnaire of general practitioners. BMC Public Health 2001;1:16. Available from: http://www.biomedcentral.com/1471-2458/1/16

This article was published on 14 May 2009.

Citation style for this article: Giovanetti F. Anaphylaxis following unnecessary meningococcal chemoprophylaxis of a healthcare worker. Euro Surveill. 2009;14(19):pii=19207. Available online: http://www.eurosurveillance.org/ViewArticle. aspx?ArticleId $=19207$ 